\title{
Cloud-based face recognition algorithm
}

\author{
Wenhan Wang \\ Nankai University College of Software \\ Kai Mu \\ Nankai University College of Software \\ Yumei Liu \\ Nankai University College of Software
}

\author{
Kehui Song \\ Nankai University College of Software \\ Junyi Deng \\ Nankai University College of Software \\ Yue Li* \\ Nankai University College of Software
}

\author{
Wei Wang* \\ Nankai University College of Software
}

\begin{abstract}
Most face recognition technology on smartphone, should deposit a photo and compare with the instant photo while validation, if the degree of similarity reaches a certain range, we consider it's his operation. However, it has many drawbacks. First, the program will take up a lot of CPU during comparison. Secondly, the pre-stored photos are easily replaced by hackers, leading to privacy information leakage. In this paper, we proposed a cloud-based identity recognition algorithm, and the standard images are stored in the Elastic Compute Service. When authenticating, our algorithm can determine whether the picture uploaded is consistent with the original picture.
\end{abstract}

Keywords: face recognition, image processing, cloud computing

\section{INTRODUCTION}

Face recognition research began in the late 1960s, and made great progress in the past 40 years, and became a hot research topic in recent years. All the domestic and foreign famous universities and institutes began to set up groups to research related subject. Face recognition subject get so much attention of the public due to its important significance, especially in both its contribution to the development of the subject and the great application prospects.

As an image pattern analyzing, understanding and classification computational subject, face recognition provide a specific issue for pattern recognition, image processing, computer vision, artificial intelligence, humancomputer interaction and other subjects .It can also be used for these subjects to try out a variety of methods and verify new theory. Intensive study of this subject can greatly promote development and maturation of these subjects.
Currently we still rely on traditional identity authentication ways to determine the input information such as ID cards (identity card, driver's license and other documents), keys (secret sign, password, etc.). However, none of these methods are convenient, safe or reliable: documents can be forged, passwords may be forgotten. These shortcomings make it increasingly hard for them to meet the needs of social development. We need a new verification technology which will give us higher reliability, security. Biometric technology is considered to be a way to solve those problems. It use the human's unique feature, such as physical characteristics or behavior, to authenticate people's identity. Physical characteristics such as face, fingerprint, retina, palm prints, DNA are also concluded. Behavior characteristics refer to the habit of behavior such as handwriting, gait, etc. As a typical biometric identification technology, face recognition technology is extremely popular with people and can be applied to all walks of life by the reason of its natural, high acceptability and other advantages.

"Eigenface" algorithm, which is the first viable human face recognition algorithm first proposed by Sirovic, Kirby, Matthew Turk and Alex Pentland using in face classification. This algorithm has a history of more than 20 years, but remains to be a typical face recognition algorithm. Consequently, any new algorithm will compare with it. In addition, there are "Fisherface", elastic graph matching techniques, partial feature matching technique from Rockefeller University, face recognition technology based on dual Bayesian probabilistic estimation and so on. Based on various algorithms people proposed, we propose a cloud face recognition algorithm based on the Android platform in this paper. 


\section{System Architecture}

This system consists of 4 modules. Acquiring image module, uploading image module, cloud image processing module and showing result module. And in order to better accomplish this function, here two separate threads are set. The main thread is responsible for interacting with the user, while the network thread is in charge of uploading images and return the JSON results after being processed.

\subsection{Acquiring Image Module}

In this module, camera will be opened after getting the user's permission. Then the image will be stored on the client as soon as the image is produced. At last, the image will be preliminary processed. For example, we can remove noise in the picture from current format like PNG, using image morphology filter and variable-threshold binarization.

\subsection{Uploading Image Module}

In this module, the picture in the first module will be uploaded to the cloud server through the input and output file stream after a connection is established via HTTPServlet and HTTPURLconnection. Then repeat steps $1-2$, and the cloud server will get two pictures.

\subsection{Cloud Image Processing Module}

This module is the core module of the system. In this system, developing an algorithm which can determines the similarity of two pictures of human face is the most important issue. In this step, the algorithm of color detection and similarity detection will be applied.

\subsection{Showing Result Module}

In this module, related operations will be done according to the result of step 3 . Then the result will be sent to the client and finally displayed to the user.

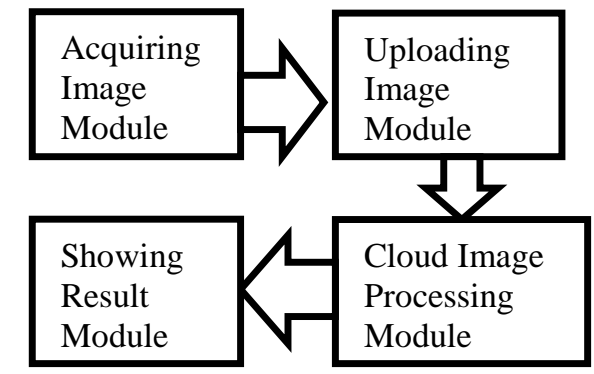

Fig. 1 the process of the system modules

\section{3. face recognition algorithm based on the cloud tech- nology}

\subsection{Image morphology filter}

Assuming that $f(x)$ is a discrete function defined on the set of $Z^{n}$, that is: $\mathrm{fi}^{\mathrm{n}} \rightarrow \mathrm{Z}$, structural element $\mathrm{B}$ is a finite subset on the set $Z^{n}$, that is $B \subset Z^{n}$. The set which is symmetric to the origin of $B$ is $B^{s}=\{-b ; b \in B\}$, the translation set of $B$ about point $x$ is
$\mathrm{B}_{\mathrm{X}}=\left\{\mathrm{b}+\mathrm{x} \mathrm{b} \in \mathrm{B}, \mathrm{x} \in \mathrm{Z}^{\mathrm{n}}\right\}$,then the two basic morphological transform definitions are as follow:

The dilation and erosion operations of $\mathrm{f}(\mathrm{x})$, s discrete form about $\mathrm{B}$ are:

$$
\begin{aligned}
& \left(f \oplus B^{s}\right)(x)=\max _{b \in B_{\lambda}}\{f(b)\} \\
& \left(f \ominus B^{5}\right)(x)=\min _{b_{b \in B_{\lambda}}\{f(b)\}}
\end{aligned}
$$

The open operation and close operation of $f(x)$ about $B$ are:

$$
\begin{aligned}
& \left(f^{\circ} B\right)(x)=\left[\left(f \ominus B^{5}\right) \oplus B\right](x)= \\
& \max _{\mathrm{a} \in B_{\mathrm{ax}}}\left\{\min _{\mathrm{b} \in \mathrm{B}_{\mathrm{a}}} \mathrm{f}(\mathrm{b})\right\} \\
& (\mathrm{f} \cdot \mathrm{B})(\mathrm{x})=\left[\left(\mathrm{f} \oplus \mathrm{B}^{5}\right) \ominus \mathrm{B}\right](\mathrm{x})= \\
& \min _{\mathrm{a} \in \mathrm{B}_{\mathrm{Bx}}}\left\{\max _{\mathrm{b} \in \mathrm{B}_{\mathrm{a}}} \mathrm{f}(\mathrm{b})\right\}
\end{aligned}
$$

\subsection{Variable threshold binarization}

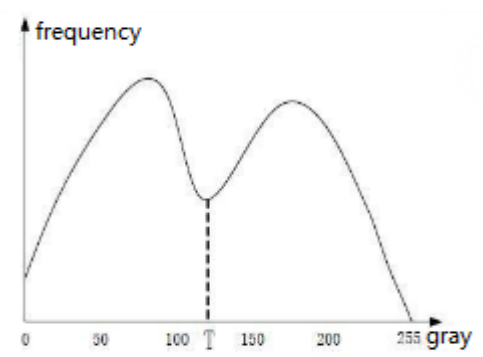

Fig. 2 graph of Variable threshold binarization[3] $g(x, y)=\left\{\begin{array}{c}255 \text { (white) } f(x, y) \geq T \\ 0(\text { black }) f(x, y)<T\end{array}\right.$

$\mathrm{f}(\mathrm{x}, \mathrm{y})$ in the formula above is gray value of gray image, $\mathrm{T}$ is the threshold value, and $\mathrm{g}(\mathrm{x}, \mathrm{y})$ is the gray image after binarization

\section{3. get location of every organ}

First ascertain the location of the eyes. Because the color of the eyes is different from around, we can adopt the method of clustering to distinguish two eyes, and then ascertain the position of the eyes according to the symmetry of face, the clustering formula is:

$V=\sum_{\mathrm{i}=1}^{\mathrm{k}} \sum_{\mathrm{x}_{\mathrm{j}} \in S_{\mathrm{i}}}\left(\mathrm{x}_{\mathrm{j}}-\mu_{\mathrm{i}}\right)^{2}$

Suppose there are $\mathrm{k}$ groups $\mathrm{Si}, \mathrm{i}=1,2, \ldots, \mathrm{k} . \mu \mathrm{i} i \mathrm{i}$ the focus of all elements $\mathrm{xj}$ in group $\mathrm{Si}$,the distance formula is Minkowski Distance formula:

$\mathrm{d}_{\mathrm{ij}}=\sqrt[x]{\sum_{\mathrm{k}=1}^{\mathrm{n}}\left|\mathrm{x}_{\mathrm{ik}}-\mathrm{x}_{\mathrm{jk}}\right|^{\lambda}}$

After ascertain the location of the eyes, let $d$ denote the distance of the eyeballs, the coordinates of the left and right eye are $\left(\mathrm{x}_{1}, \mathrm{y}_{1}\right),\left(\mathrm{x}_{2}, \mathrm{y}_{2}\right)$, then the region of the organ is ascertained as follow:

Left eye: 
$\left(\mathrm{x}_{1}-\mathrm{e}_{1}, \mathrm{y}_{1}-\mathrm{e}_{2}, \mathrm{x}_{1}+\mathrm{e}_{2}, \mathrm{y}_{1}+\mathrm{e}_{4}\right)$

Right eye:

$\left(\mathrm{x}_{2}-\mathrm{e}_{1}, \mathrm{y}_{2}-\mathrm{e}_{2}, \mathrm{x}_{2}+\mathrm{e}_{\mathrm{a}}, \mathrm{y}_{2}+\mathrm{e}_{4}\right)$

Let the displacement be:

$e_{1}=e_{2}=0.4 d, e_{2}=e_{4}=0.5 d$,

Nose:

$\left(\frac{\mathrm{x}_{1}+\mathrm{x}_{2}}{2}-\mathrm{n}_{1}, \frac{\mathrm{y}_{1}+\mathrm{y}_{2}}{2}+\mathrm{c}_{1} \mathrm{~d}-\mathrm{n}_{2}, \frac{\mathrm{x}_{1}+\mathrm{x}_{2}}{2}+\right.$

$\left.\mathrm{n}_{\mathrm{a}}, \frac{\mathrm{y}_{1}+\mathrm{y}_{2}}{2}+\mathrm{c}_{1} \mathrm{~d}+\mathrm{n}_{4}\right)$

Let the displacement be:

$\mathrm{n}_{1}=\mathrm{n}_{\mathrm{a}}=0.5 \mathrm{~d}, \mathrm{n}_{2}=\mathrm{n}_{4}=0.5 \mathrm{~d}$,

The coefficient is:

$\mathrm{c}_{1}=0.9$

Mouse:

$\left(\frac{\mathrm{x}_{1}+\mathrm{x}_{2}}{2}-\mathrm{m}_{1}, \frac{\mathrm{y}_{1}+\mathrm{y}_{2}}{2}+\mathrm{c}_{2} \mathrm{~d}-\mathrm{m}_{2}, \frac{\mathrm{x}_{1}+\mathrm{x}_{2}}{2}+\right.$

$\left.\mathrm{m}_{\mathrm{a}}, \frac{\mathrm{y}_{1}+\mathrm{y}_{2}}{2}+\mathrm{c}_{2} \mathrm{~d}+\mathrm{m}_{4}\right)$

Let the displacement be:

$\mathrm{m}_{1}=\mathrm{m}_{\mathrm{a}}=0.7 \mathrm{~d}, \mathrm{~m}_{2}=\mathrm{m}_{4}=0.4 \mathrm{~d}_{s}$

The coefficient is:

$c_{2}=1.5$

Finally we can figure out that two faces are different by comparing feature vector weighted similarity.

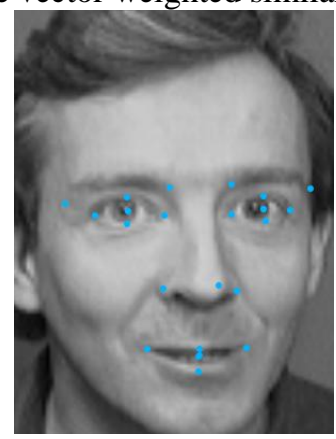

Fig. 3 picture on how to locate different organs

\subsection{Experiment One:}

Do an experiment of 40 faces using the ORL face database (five faces of each people)
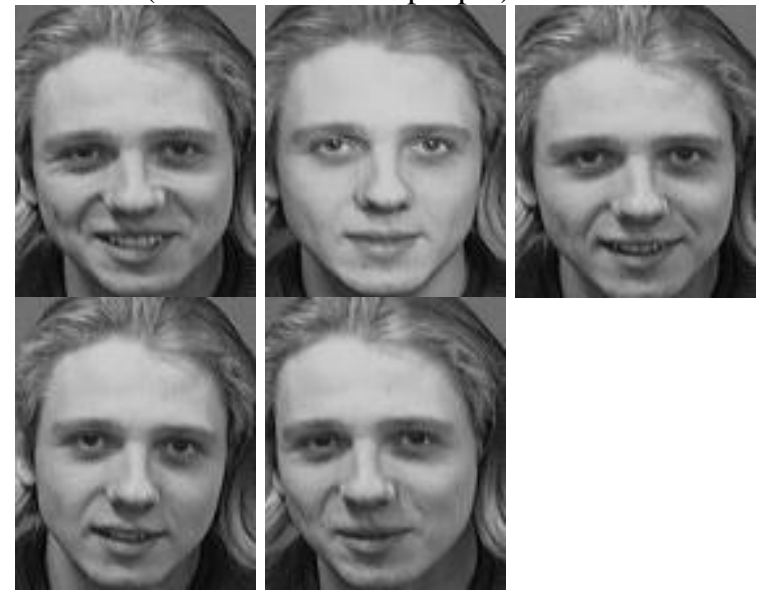

Fig. 4 five different photos of one person

Measure the similarity of the latter four faces and the first face, results are shown below:
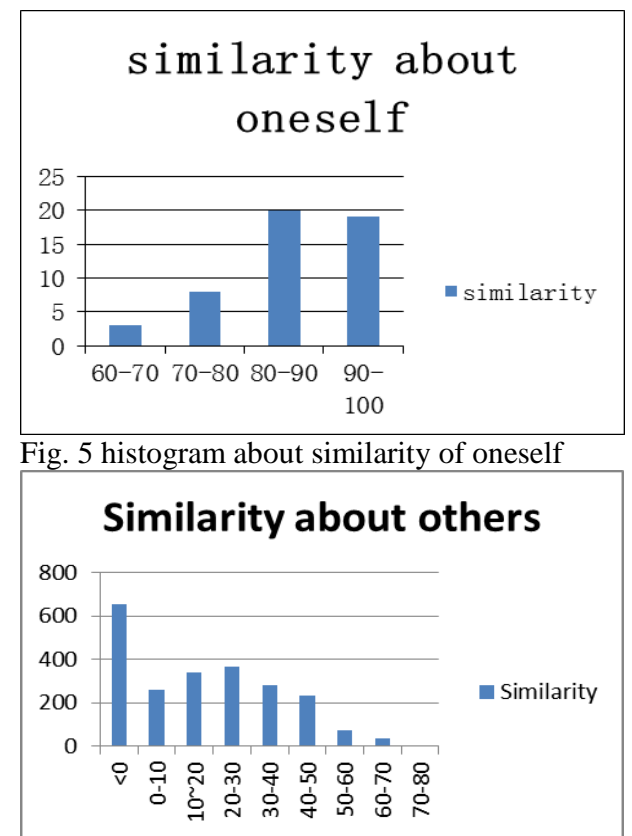

Fig. 6 histogram about similarity of others

The situation in which the similarity of determining the two faces are of one person is 86 causes the least possibility of miscalculation.

\subsection{Experiment Two:}

After test, in different light conditions, the algorithm still has high identification, which illustrates the recklessness of the algorithm.

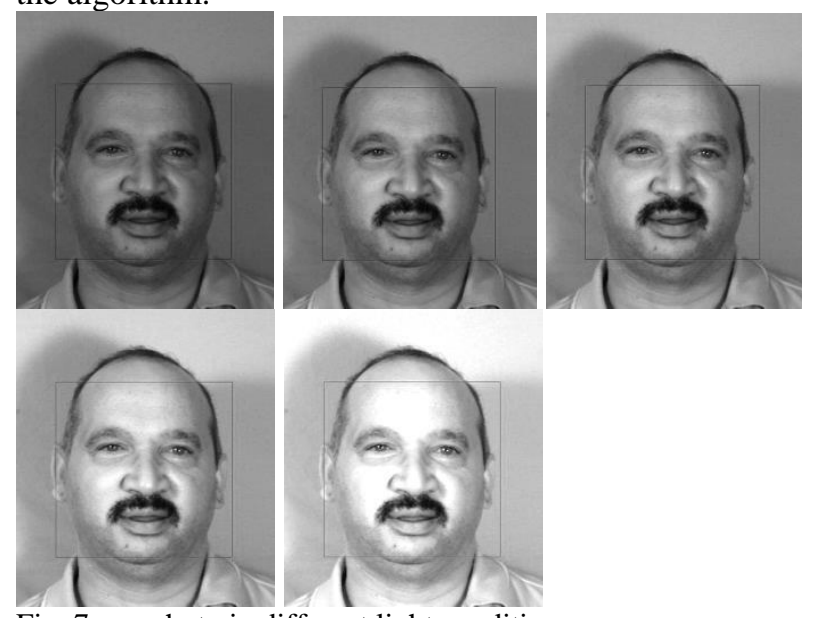

Fig. 7 one photo in different light conditions

Table 1.The result of second experiment compared to the photo at 12:00

\begin{tabular}{|c|c|}
\hline Time & similarity \\
\hline $06: 00$ & 0.912279 \\
\hline $08: 00$ & 0.861503 \\
\hline $14: 00$ & 0.898528 \\
\hline $16: 00$ & 0.932675 \\
\hline $18: 00$ & 0.933902 \\
\hline
\end{tabular}




\section{Conclusion}

For the emerging privacy leakage problem, this paper mainly puts forward the smartphone face recognition method, which is different from the traditional password protection. Our face recognition method is more convenient, and users' acceptability is also higher. Our algorithm mainly uses the image morphology filtering and variable threshold binarization method, which can protect users' privacy effectively. With this system, it will be more reliable and convenient to enter some software which require authentication.

\section{References}

[1] Zhang Jun, He Xin, Li Jieg u. Face recognition based on geometrical feature points extraction. China Academic Journal Electronic Publishing House. 1999:40-43.

[2] He Qing-hang, He Ji-shan. The Smoothing of Gray Image Based on Morphological Filtration. China Academic Journal Electronic Publishing House. 2004:58-60

[3] http://wenku.baidu.com/view/52c113650b1c59eef8c7b4 f6.html 